\title{
Adenocarcinoma in Tubular Adenoma
}

National Cancer Institute

\section{Source}

National Cancer Institute. Adenocarcinoma in Tubular Adenoma. NCI Thesaurus. Code C7677.

An adenocarcinoma arising from a tubular adenoma. 